\title{
NATIONAL INSTITUTE FOR FUSION SCIENCE
}

Turbulence and Transport Characteristics of a Barrier in a Toroidal Plasma

A. Fujisawa, A. Shimizu, H. Nakano, S. Ohsima, K. Itoh, H. Iguchi, Y. Yoshimura, T. Minami, K. Nagaoka, C. Takahashi, M. Kojima, S. Nishimura, M. Isobe, C. Suzuki, T Akiyama, Y. Nagashima,

K. Ida, K. Toi, T. Ido, S.-I. Itoh, K. Matsuoka, and S. Okamura

(Received - Sep. 26, 2005) 
Inquiries about copyright should be addressed to the Research Information Center, National Institute for Fusion Science, Oroshi-cho, Toki-shi, Gifu-ken 509-5292 Japan.

E-mail: bunken@nifs.ac.jp

\section{$<$ Notice about photocopying $>$}

In order to photocopy any work from this publication, you or your organization must obtain permission from the following organizaion which has been delegated for copyright for clearance by the copyright owner of this publication.

\section{Except in the USA}

Japan Academic Association for Copyright Clearance (JAACC)

6-41 Akasaka 9-chome, Minato-ku, Tokyo 107-0052 Japan

Phone: 81-3-3475-5618 FAX: 81-3-3475-5619 E-mail: jaacc@ mtd.biglobe.ne.jp

In the USA

Copyright Clearance Center, Inc.

222 Rosewood Drive, Danvers, MA 01923 USA

Phone: 1-978-750-8400 FAX: 1-978-646-8600 


\title{
Turbulence and transport characteristics of a barrier in a toroidal plasma
}

\author{
A. Fujisawa, A. Shimizu, H. Nakano, S. Ohsima, K. Itoh, \\ H. Iguchi, Y. Yoshimura, T. Minami, K. Nagaoka, C. \\ Takahashi, M. Kojima, S. Nishimura, M. Isobe, C. Suzuki, \\ T Akiyama, Y. Nagashima ${ }^{1}$, K. Ida, K. Toi, T. Ido, S.-I. \\ Itoh $^{1}$, K. Matsuoka, and S. Okamura \\ National Institute for Fusion Science, Oroshi-cho, Toki-shi, 509-52 Japan \\ ${ }^{1}$ RIAM, Kyushu Univ., Kasuga 816 Japan
}

\begin{abstract}
Turbulence and zonal flow at a transport barrier are studied with twin heavy ion beam probes in a toroidal helical plasma. A wavelet analysis is used to extract turbulence properties, $e$. $g$., spectra of both density and potential fluctuations, coherence and phase between them, and the dispersion relation. Particle transport estimated from the fundamental characteristics is found to clearly rise with their intermittent activities after the barrier is broken down. The time-dependent analysis reveals that intermittency of turbulence is correlated with evolution of stationary zonal flow.
\end{abstract}

Keywords; internal transport barrier, turbulence, zonal flow, particle flux, wavelet analysis, heavy ion beam probes 


\section{Introduction}

Structural formation related to turbulence is ubiquitous in nature and the universe[1]. The examples include swirling pipe flow, accretion disks around back-holes, Jovian belt and solar tachocline[2], as well as structure of laboratory plasmas. The research oriented to realizing a fusion reactor has revealed that the turbulence governs transport and often leads to structural bifurcation in toroidal plasmas.

The first demonstration of the bifurcation property was the H-mode in ASDEX[3], being followed by findings of other bifurcated states, for example, families of edge and internal transport barriers, and others[4]. The discoveries have stimulated interests in transport and bifurcation mechanisms of toroidal plasmas. At present it is widely known that the cause of the phenomena can be ascribed to relationship between sheared radial electric field (or flow perpendicular to magnetic field) and turbulence. Shear structure in radial electric field, that is possibly caused by a bifurcation (or nonlinear) nature of mean flow [5,6] or turbulent Reynolds stress[7], can control the turbulence, transport, and resultant structure[8].

Recent simulation and theoretical works have suggested that the meso-scopic structure, termed zonal flows $[9,10,11,12,13,14]$, should play an important role in the structural formation in a toroidal plasma through a nonlinear interaction with turbulence. The presence of stationary zonal flow, in fact, has been just confirmed experimentally with dual heavy ion beam probes (HIBP) in Compact Helical System (CHS)[15]. This trend means that the third element, zonal flow, is newly coming up for physics of transport and structural formation in turbulent plasmas.

An internal transport barrier, that was found in CHS for the first time[16], becomes common in toroidal helical plasmas[17, 18, 19]. In a recent CHS experiment, the dual HIBPs succeeded to catch an exact moment of the back- transition. This article reports the observation of turbulence properties at the barrier position, before and after the transition, including mean electric field, and zonal flows. The resultant particle flux is estimated with time-dependent manner using a wavelet analysis. The analysis demonstrates a causal relationship between zonal flow component and turbulence in the state with the transport barrier.

\section{Experimental set-up and wavelet analysis}

The stage of the experiments, CHS, is a toroidal helical device, of which the major and averaged minor radii are $R=1 \mathrm{~m}$ and $a=0.2 \mathrm{~m}$, respectively. Each of the HIBPs is equipped with three channels that can observe the adjacent spatial points of the plasma. The HIBP is a synthetic diagnostics to measure local density, potential, (possibly magnetic field) and their fluctuations simultaneously[20, 21]. The energy difference between the injected and detected beams corresponds to the plasma potential at the ionization point. The fluctuation in detected beam intensity could give information of local density fluctuation at the ionization point.

The target plasma for the experiments was sustained with electron cyclotron resonance heating of $\sim 200 \mathrm{~kW}$. The magnetic field strength of the discharges was $B=0.88 \mathrm{~T}$, and the density was constantly maintained at $n_{\mathrm{e}} \simeq 5 \times 10^{12} \mathrm{~cm}^{-3}$. The twin HIBP measurements were performed in the core region of the plasma, where the electron temperature $(\sim 1 \mathrm{keV})$ was sufficiently high for the ionization cross-section to be insensitive to the temperature, and the electron density was sufficiently low to neglect the integrated density fluctuation along the beam orbit. Therefore, the change 
in the current should mainly reflect the local density. The band-width of the amplifiers to detect beam current was set to $250 \mathrm{kHz}$, corresponding to the Nyquist frequecy in the sampling rate of $2 \mu \mathrm{s}$.

An analysis using the Morlet's (or Milligen's) wavelet[23] is performed on the signals of the barrier in order to examine temporal evolution of turbulencedriven particle flux. The wavelet is a natural extension of the traditional Fourier transformation. The definition of the wavelet transformation is $\Phi(f, t)=$ $\sqrt{f} \exp \left[i 2 \pi f t-(f t)^{2} / 2\right]$, with $F(f, t)=\int_{-\infty}^{\infty} f(\tau) \Phi(f, t-\tau) d \tau$. The auto-power and cross-power are calculated as $P_{\text {auto }}(F ; f, t)=(2 T)^{-1} \int_{t-T}^{t+T} F(f, \tau) F^{*}(f, \tau) d \tau$ and $P_{\text {crs }}(F, G ; f, t)=(2 T)^{-1} \int_{t-T}^{t+T} F(f, \tau) G^{*}(f, \tau) d \tau$, respectively. Hence, the calculated power represents a temporal average in the period of $2 T$. Here, the period of the window $T$ is chosen to be $0.25 \mathrm{~ms}$ and the integral is simplified to summation of discrete time $\tau_{i}$ in the actual analysis. The interval of neighboring ensembles, being different from each frequency, is chosen as $\Delta \tau_{i}=1 / 2 f$. In the present analysis, the wavelet spectra are calculated from 5 to $200 \mathrm{kHz}$ in every $5 \mathrm{kHz}$. Note that the uncertainty principle of $\Delta f \Delta t \sim 1$ gives a constraint on the resolution of time and freqeuncny.

\section{Difference of fluctuation characteristics before and after transition}

Figure 1 shows the discharge to exhibit transition from the state with a barrier to the one without the barrier; here we refer to the former and the latter as H- and L-phase, respectively. The solid lines represent the potential waveforms of the center channels of the two HIBPs. The observation point of the first HIBP is located by $\sim 1.5 \mathrm{~cm}$ inside the barrier, while the observation point of the second HIBP is located at the exact barrier foot-point $(\mathrm{r}=6.4 \mathrm{~cm} / \rho=0.34)$. The first HIBP potential clearly shows transition at $t=71.3 \mathrm{~ms}$, that evolves in a time scale of less than a hundred micro seconds due to neoclassical characteristics[22]. On the other hand, the other potential at the barrier shows no significant change.

Figure 2a shows the ensemble-averaged wavelet spectra for the windows for Land $\mathrm{H}$ - phases. The increase in the fluctuation power in the L-phase is clear in both density and potential, particularly in the frequency from $\sim 50$ to $\sim 100 \mathrm{kHz}$. It has been already known that the fluctuation reduction at the barrier should result from the $E \times B$-shearing caused by the neoclassical bifurcation of radial electric field[16]. The attached bars represent the degree of temporal variation, or the standard deviation of power density. For comparison, the spectra using the Fast Fourier Transformation (FFT) are shown by the thin solid lines. These two spectra show a practically good agreement, although the base of the wavelet is not orthogonal.

Figure $2 \mathrm{~b}$ shows the ensemble averages of the phase and coherence between density and potential fluctuations for the L- and H-phases. Here, the phase difference and coherence are evaluated from the cross-spectrum in a time-dependent manner. The phase $\theta(n, \phi ; t)$ and coherence $\gamma(n, \phi ; t)$ are obtained from the relations, $P_{\text {crs }}(n, \phi ; t)=$ $\left|P_{\text {crs }}(n, \phi ; t)\right| e^{i \theta(n, \phi ; t)}$ and $\gamma(n, \phi ; t)=\left|P_{\text {crs }}(n, \phi ; t)\right| / \sqrt{P_{\text {auto }}(n ; t) P_{\text {auto }}(\phi, t)}$. The bars in Fig. 2b represent the standard deviation of the phase and coherence. In both states, the phase increases monotonically below $\sim 50 \mathrm{kHz}$, and becomes a constant value above $\sim 50 \mathrm{kHz}$. Here, the positive phase shift means that potential fluctuation is ahead of density fluctuation by $\sim \pi / 2$, indicating interchange-like characteristics. The coherence shows a high value $(>0.4)$ in the frequency below $\sim 150 \mathrm{kHz}$. 
In the measurements of HIBP, the wave number can be evaluated by dividing the phase difference of adjacent observation points by their distance of $\sim 5 \mathrm{~mm}$. Figure 3 shows the ensemble-averaged coherence and dispersion obtained from adjacent potential signals of center and inner channels for both phases. In both phases, the wave number increases below $\sim 120 \mathrm{kHz}$. The positive phase difference signifies that the wave should propagate in the electron diamagnetic direction for the L- and $\mathrm{H}$ phases. Above $\sim 120 \mathrm{kHz}$, the coherence should be too low to estimate significant phase difference. The uncertainty of the wave number in the frequency range, however, gives an error of less than $1 \%$ to the total absolute particle flux, owing to negligible fluctuation power in the frequency range.

The particle flux density can be calculated in the form of $d \Gamma_{\text {turb }}(f, t) / d f=$ $B^{-1} k(f, t) \operatorname{Im}\left(P_{\text {crs }}(n, \phi ; t)\right)=B^{-1} k(f, t) \sqrt{P_{n}(f, t) P_{\phi}(f, t)} \gamma(f, t) \sin \delta(f, t)$, where $\operatorname{Im}$ and $k(f)$ mean the extraction of the imaginary part and the wave number, respectively. The formula is an extension of particle flux estimation using the traditional Fourier analysis[24]. Figure 4a shows an image plot of particle flux density, particle flux density at $60 \mathrm{kHz}$ and the total particle flux integrated over all frequency up to $200 \mathrm{kHz}$. These signals show intermittent bursts $[25,26]$, and the averaged value and intensity of the bursts increase in L-phase. The ordinary bursts orient radially outward or poloidally in the ion diamagnetic direction, while occasional bursts occur in the opposite direction. The image plot indicates that the particle flux density from $\sim 50 \mathrm{kHz}$ to $\sim 100 \mathrm{kHz}$ has the most dominant contribution to the particle transport in both L- and $\mathrm{H}$-phases.

In Fig. 4b, probability density functions (PDF) are plotted to characterize the intermittent activity of the estimated particle fluxes in L- and H-phases. Table 1 shows the moments of the PDFs; average $(\mu)$, variance $(\sigma)$, skewness (S), and kurtosis $(\mathrm{K})$. Obviously, the average and variance manifest the increases in transport and burst intensity in L-phase, respectively. Besides, the enhancement in skewness and kurtosis in the L-phase indicates a deviation of particle transport from the Gaussian characteristics $(S=0$ and $K=3$ ) or nonlinear activity increases in the L-phase.

\section{Correlation between zonal flow and turbulence}

Similarly to the previous identification of zonal flow, the twin HIBPs system confirmed quite high coherence $(\sim 0.7)$ between electric fields at two toroidal locations in the frequency below $\sim 1 \mathrm{kHz}$ for L- and $\mathrm{H}$-phases in the present series of discharges. Figure 5 shows a comparison between temporal patterns of the particle flux density (a part of Fig. 4a) and the zonal flow or low pass filtered potential difference between the inner and center channels[15]. Obviously, the zonal flow amplitude decreases after the transition. The real electric field (or flow velocity) can be estimated from the potential difference divided by channel separation $(\sim 5 \mathrm{~mm})$. Here, the positive value means that the zonal flow increases in ion diamagnetic direction, in other words, total flow intensity increases since the mean flow is in the ion diamagnetic direction (electric field is positive).

An important finding from the comparison is that similar activities could be seen in the patterns of zonal flow and particle flux density. In order to quantify the correlation of turbulence and zonal flow, the wavelet spectra are evaluated for periods discriminated by the phase of the zonal flow. Figure $5 \mathrm{~b}$ shows the density and potential spectra obtained in the above manner for the $\mathrm{H}$-phase, since the larger amplitude of zonal flow in the H-phase easily differentiate the phase of zonal flow. Around $\sim 50$ $\mathrm{kHz}$ and $\sim 120 \mathrm{kHz}$, the fluctuation power in the minimum phase of zonal flow is 
stronger than that in the maximum phase of zonal flow. In addition, the power at the intermediate (or zero) phase shows the mean value of the two. The result, therefore, substantiates a causal relationship between zonal flow and the turbulence.

\section{Discussion and Summary}

Several processes can be counted up to explain the causal link between the turbulence and zonal flow [9]; i) quasi-energy conservation between turbulence and zonal flow, ii) shearing of zonal flow on the turbulence, and iii) turbulent wave trapping of zonal flows. If the turbulent waves are trapped in a zonal flow valley, the turbulence structure should move together with a change in a local pattern of zonal flow. The present experimental accuracy, however, cannot distinguish which process is most dominant to cause such a relationship, since two point measurements of electric field is too coarse to deduce the detailed structure of zonal flow and the incident shearing rate.

In summary, temporal behaviors in turbulence and resultant particle flux are successfully evaluated using a wavelet analysis at the barrier foot-point in the states with and without a transport barrier. The analysis shows the following points; i) the turbulence, resultant transport and their intermittency increase after the transport barrier is broken down, ii) the turbulence of $50-100 \mathrm{kHz}$ is the major fluctuation to drive particle transport in both states. iii) the enhanced transport without a barrier can be ascribed to an increase in fluctuation power in this frequency range, since the dispersion relation and the phase between density and potential fluctuations show no significant change. Finally, in the state with the barrier it is confirmed that the zonal flow should really give an impact on or modulation of turbulence and turbulence-driven flux density through a causal link. The observation is the first experimental evidence to verify a relationship of stationary zonal flow with the turbulence and resultant transport.

This work is partly supported by the Grant-in-Aids for Scientific Research (No. 15360497) and Specially-Promoted Research (No.16002005). The authors are grateful to Prof. O. Motojima for his continuous supports and encouragements.

[1] A. Yoshizawa, S. -I. Itoh, K. Itoh, Plasma and Fluid Turbulence (Institute of Phys. Pub., Bristol and Philadelphia, 2003).

[2] S. -I. Itoh, K. Itoh, A. Yoshizawa, N. Yokoi, Astrophy. Jour. 6181044 (2005).

[3] F. Wagner et al., Phys. Rev. Lett. 491408 (1982).

[4] A. Fujisawa, Plasma Phys. Control. Fusion 45 R1 (2003).

[5] S. -I. Itoh, K. Itoh, Phys. Rev. Lett. 602276 (1988)

[6] K. C. Shaing, E. C. Crume Jr., Phys. Rev. Lett., 632369 (1989).

[7] P. H. Diamond, Y. -M. Liang, B. A. Carreras, P. W. Terry, Phys. Rev. Lett. 722565 (1994).

[8] H. Biglari, P. H. Diamond, P. W. Terry, Phys. Fluids B 21 (1990); K. Itoh, S.-I. Itoh, A. Fukuyama, H. Sanuki, M. Yagi,Plasm Phys. Control. Fusion 36123 (1994).

[9] P. H. Diamond, K. Itoh, S.-I. Itoh, T. S. Hahm, Plasma Phys. Control. Fusion 47 R35 (2005).

[10] P. H. Diamond, et al. in Proceedings of the 17th IAEA fusion energy conference, Yokohama, 1998 vol. 4 1421-1428. (International Atomic Energy Agency, Vienna, Austria, 1999).

[11] N. Winsor, J. L. Johnson, and J. M. Dawson, Phys. Fluids 11, 2448 (1968).

[12] K. Hallatschek, D. Biskamp, Phys. Rev. Lett. 861223 (2001).

[13] M. N. Rosenbluth, F. L. Hinton, Phys. Rev. Lett. 80, 724 (1998).

[14] Z. Lin, T. S. Hahm, W. W. Lee, W. M. Tang, R. B. White, Science 281, 1835 (1998).

[15] A. Fujisawa et al., Phys. Rev. Lett. 93165002 (2004).

[16] A. Fujisawa, et al. Phys. Rev. Lett. 82, 2669(1999).

[17] U. Stroth, K. Itoh, S. -I. Itoh, H. Hartfuss, H. Laqua, Phys. Rev. Lett. 865910 (2001). 
[18] F. Castejón, V. Tribaldos, I. García-Cortes, E. de la Luna, J. Herranz, I. Pastor, T. Estrada, TJ-II Team, Nucl. Fusion 42271 (2002).

[19] K. Ida et al., Phys. Rev. Lett., 91085003 (2003).

[20] A. Fujisawa, et al. Rev. Sci. Instrum. 67, 3099 (1996).

[21] T. P. Crowley, IEEE Trans. Plasma Sci. 22291 (1994).

[22] A. Fujisawa, et al. Phys. Rev. Lett. 79, 1054 (1997).

[23] B. Ph. van Milligen, C. Hidalgo, E. Sanchez, Phys. Rev. Lett. 74 395(1995).

[24] E. J. Powers, Nucl. Fusion 14749 (1974).

[25] M. Endler, H. Nieedermeyer, L. Giannone, L. Holzhauer, E. Rudyj, G. Theimer, N. Tsois, ASDEX team, Nucl. Fusion 351307 (1995).

[26] J. Castellano et al., Phys. Plasmas 9713 (2002). 
Figure 1. Transition in potential measured with heavy ion beam probes. A potential signal inside the barrier shows an exact time of back-transition. The other signal is the potential exactly at a barrier foot-point, $\rho \sim 0.34$. The waveform around the transition is roughly expressed as $\phi \sim \tanh \left[\left(t-t_{0}\right) / \alpha\right]$ with $t_{0}=71.3 \mathrm{~ms}$ and $\alpha=0.2 \mathrm{~ms}$.

Figure 2. (a) Ensemble-averaged wavelet spectra of density and potential fluctuations in L- and H-phases. (b) Coherence and phase between density and potential fluctuations in L- and H-phases.

Figure 3. Dispersion relationship and coherence etimated from the phase difference between potential fluctuations of adjacent two channels.

Figure 4. (a) An image plot of temporal evolution of estimated particle flux density, the waveforms of a particle flux density at $60 \mathrm{kHz}$ and the total particle flux. (b) Probability density functions of total flux and flux density at $60 \mathrm{kHz}$. Here, the flux and the flux density are normalized by $1 \times 10^{20} \mathrm{~m}^{-2} s^{-1}$. and $1 \times 10^{16} \mathrm{~m}^{-2}$, respectively.

Figure 5. A causal relationship between turbulence and zonal flow. (a) Temporal evolution of zonal flows with an image plot of particle flux (a portion of Fig. 4a for the range of $30-110 \mathrm{kHz}$ ). (b) Density and (c) potential fluctuation spectra in the time windows discriminated by zonal flow phase; $i$. e., maxima, zero and minima.

Table 1. Characteristic values of PDFs for total particle flux and flux density at $60 \mathrm{kHz}$ for L- and H-phases; $\mu, \sigma, S$ and $K$ represent average, variance, skewness, and kurtosis, respectively. Here, $\mu$ and $\sigma$ are normalized by $1 \times 10^{20} \mathrm{~m}^{-2} s^{-1}$ and $1 \times 10^{16} \mathrm{~m}^{-2}$ for the total particle flux and the particle density, respectively.

\begin{tabular}{lllll}
\hline & $\mu$ & $\sigma$ & $\mathrm{S}$ & $\mathrm{K}$ \\
\hline H-phase (total) & 0.13 & 0.21 & 0.20 & 4.5 \\
L-phase (total) & 0.75 & 0.57 & 0.48 & 6.3 \\
H-phase $(60 \mathrm{kHz})$ & 0.045 & 0.05 & 0.51 & 5.1 \\
L-phase $(60 \mathrm{kHz})$ & 0.17 & 0.20 & 0.85 & 7.2 \\
\hline
\end{tabular}




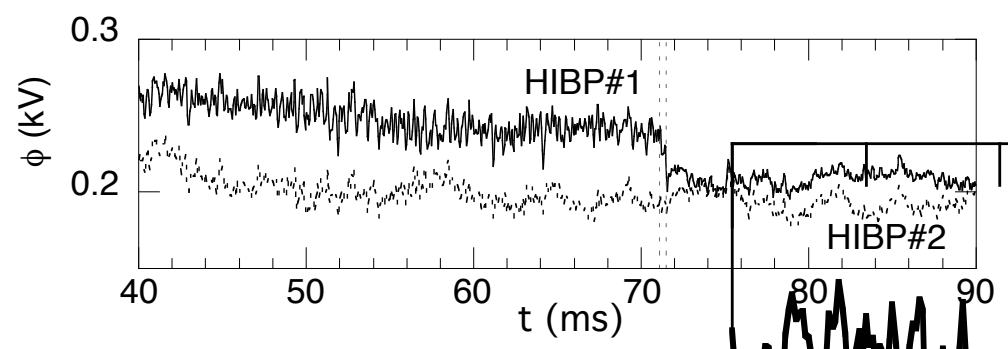

Figure 1. A. Fujisawa et al 
(a)
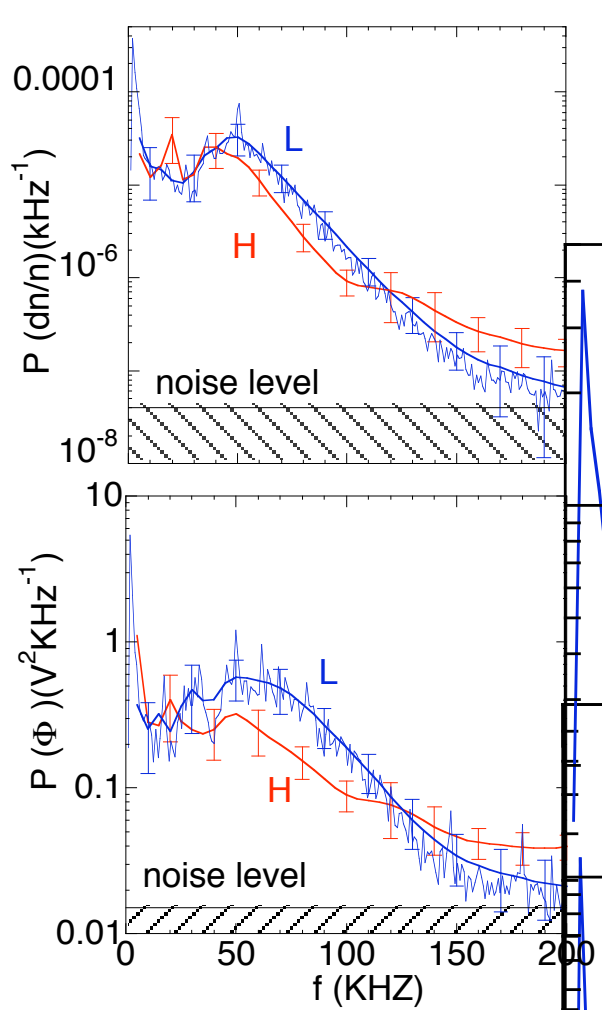

(b)
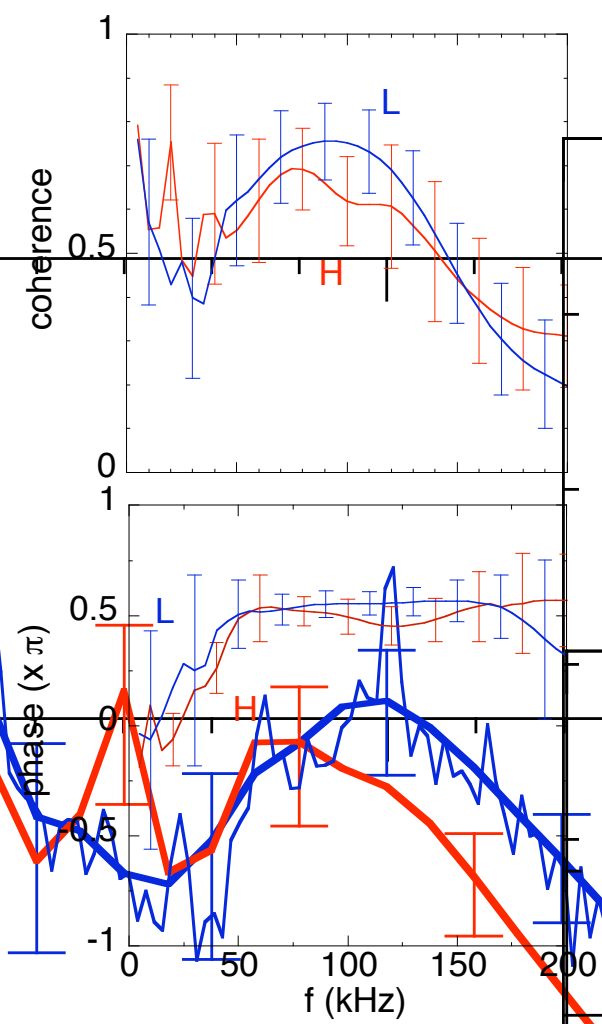

Figure 2. A. Fujisawa et al 

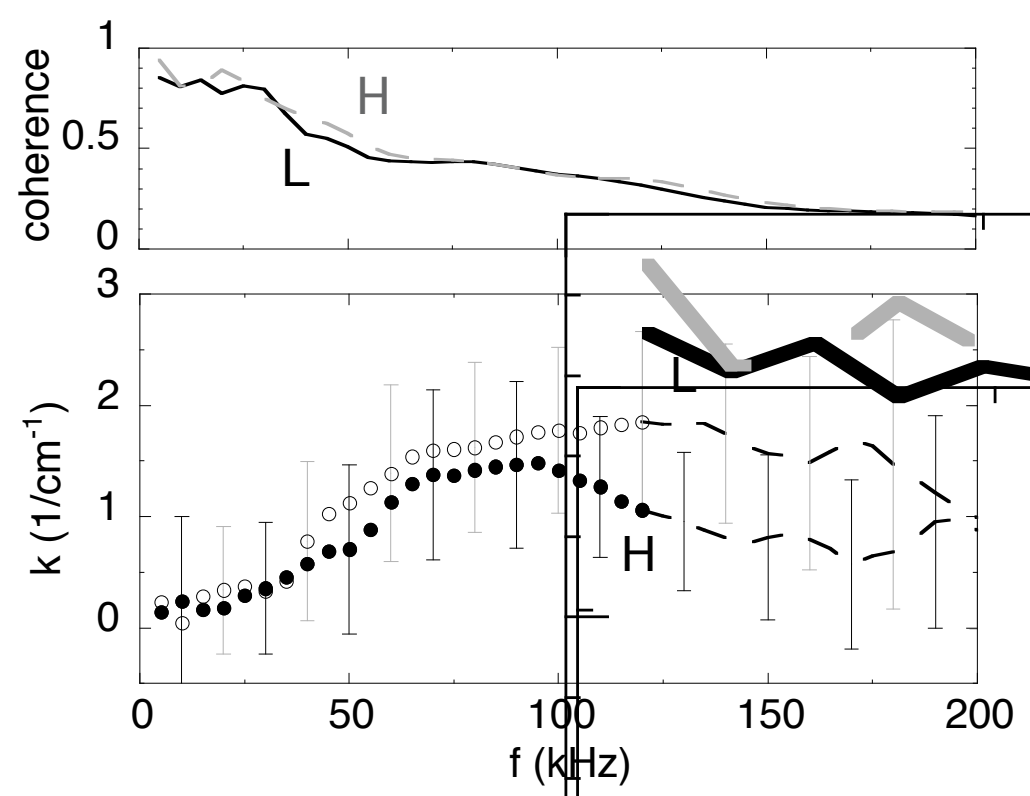

Figure 3. A. Fujisawa et al 
(a)

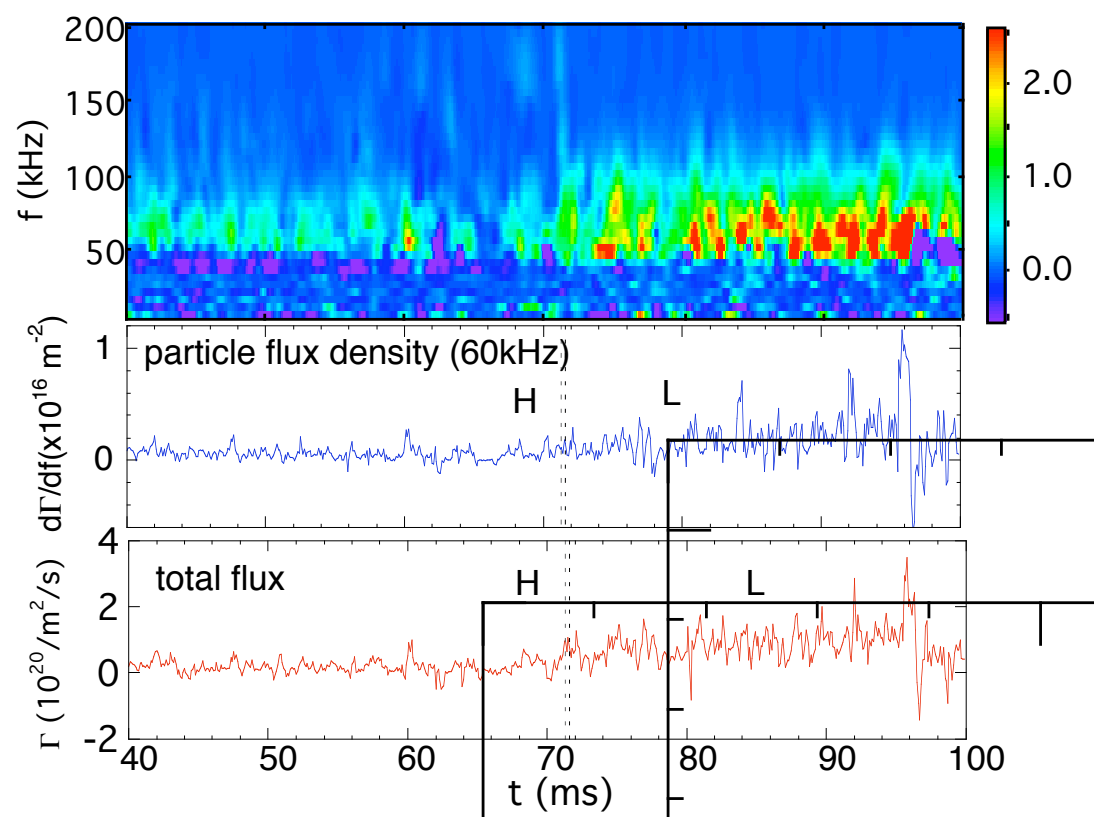

(b)
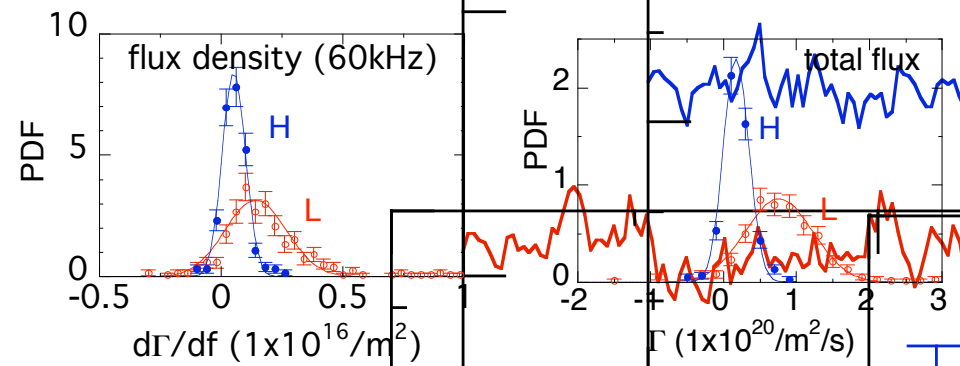

Figure 4. A. Fujisawé 
(a)

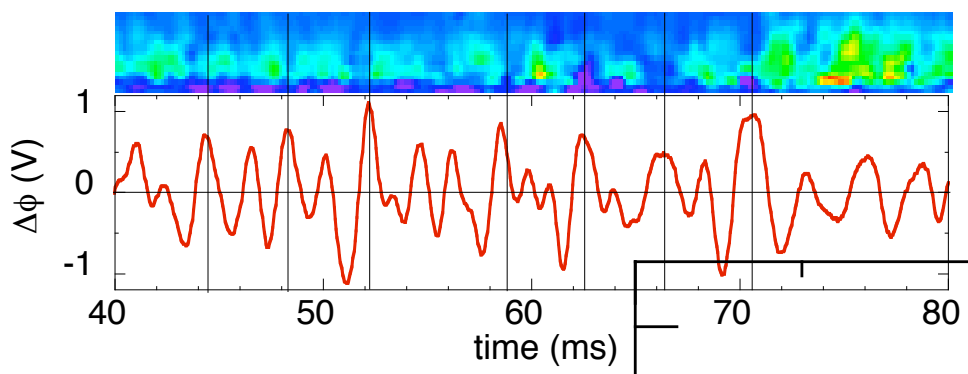

(b)

(c)
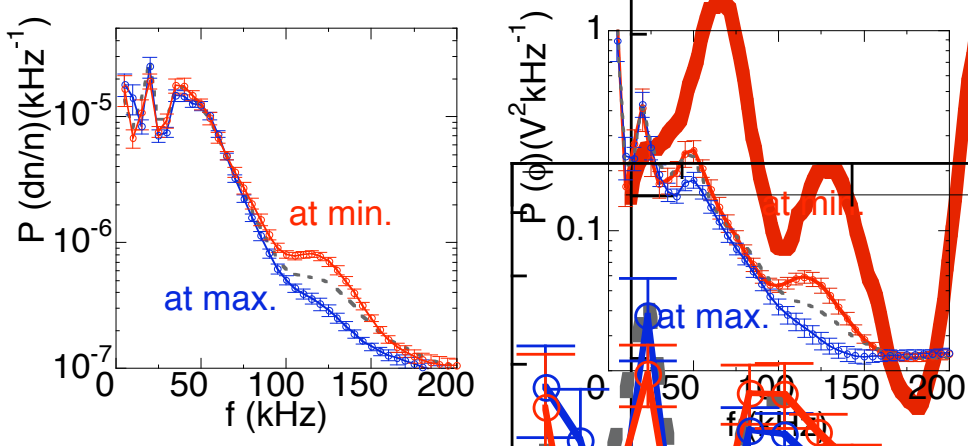

Figure 5. A. Fujisawa et al 